\title{
Detection of MRSA nasal carriage in hospital population using chromagar
}

\begin{abstract}
Background: Methicillin-resistant Staphylococcus aureus (MRSA) is an important human pathogen and normally colonized in body parts including skin, nose, inguinal folds, perineum and throat. It accounts for nearly two-third of all S. aureus infections in hospitals. MRSA is resistant not only to all $\beta$-lactam groups but also other antibiotics including aminoglycosides, tetracycline and macrolides. The study was undertaken for the rapid detection of MRSA utilizing CHROMagar MRSA (CMRSA) at Department of Microbiology, University of Health Sciences, Lahore for the period of six months.
\end{abstract}

Methods: Two hundred nasal samples were taken from 3 days old hospitalized subjects with sterile wooden swab, inoculated on CMRSA and Mueller Hinton agar (MHA) with cefoxitin disc $(30 \mu \mathrm{g})$. After $24 \mathrm{hr}$, the colonies were identified on both the agar plates which were then further reincubated for the next $48 \mathrm{hr}$.

Results: At $37^{\circ} \mathrm{C}$ of incubation after $24 \mathrm{hr}, 10.5 \%$ of the total studied samples were positive for MRSA while another $12 \%$ samples showed positive results with an extended period of incubation upto $48 \mathrm{hr}$ on CMRSA plates. Thus a total of $22.5 \%$ were positive for MRSA. $6.5 \%$ of the total samples showed methicillin resistance confirmed with novobicin disc $(30 \mu \mathrm{g})$ and were identified as Staphyloccocus epidermidis (MRSE) on MHA with cefoxitin disc after 24h of incubation.

Conclusion: It is concluded that CMRSA is equal in activity as compared to Mueller Hinton agar with cefoxitin disc which requires at least 3 days of sample processing, so CMRSA can also be used for the rapid detection of MRSA without utilization of additional sources.

Keywords: staphyloccocus aureus, beta-lactam, methicillin-resistant, staphylococcus aureus (MRSA), staphyloccocus epidermidis, CHROMagar MRSA, mueller hinton agar, cefoxitin, FOX
Volume 2 Issue 4 - 2017

\author{
Sidrah Saleem,' Sana Fatima,' Muddasir \\ Hassan $\mathrm{Abbasi}^{2}$ \\ 'University of Health Sciences, Pakistan \\ ${ }^{2}$ Department of Zoology, University of the Punjab, Pakistan
}

Correspondence: Sana Fatima, University of Health Sciences, Pakistan, Email sf.koolsun2I@gmail.com

Received: September 08, 2017 | Published: December 29, 2017

\section{Introduction}

Methicillin resistant Staphylococcus aureus (MRSA) is a human pathogen that is capable of causing infections of skin and soft tissue, surgical site, respiratory and urinary tract that may results into severe morbidity and mortality. ${ }^{1,2}$ It is resistant to all $\beta$ lactam and other antibiotics like aminoglycosides, tetracycline, macrolides etc. ${ }^{3}$ The most important reservoir for its spread are symptomatic/ asymptomatic patients and healthcare workers that carry MRSA in their anterior nares. ${ }^{4,5}$ Asymptomatic colonization with it leads to high risk of its subsequent infection not only for carrier but to other patients as well. ${ }^{6}$ Worldwide, nasal carriage is recommended for screening of MRSA for its appropriate antibiotic selection and timely treatment of asymptomatic carriers. ${ }^{7}$ In Pakistan, a study was done in 2015 in Abbottabad in which MRSA was found to be $70.8 \%$ in the total samples. ${ }^{8}$ Screening for MRSA is done employing various methods including enriched media, differential solid media and broth culture enhancement media.

Several studies have utilized phenotypic and genotypic methods for rapid identification of MRSA, including direct identification, susceptibility testing, DNA probes, real-time PCR, and immunologic approaches. Each method has its own advantages and disadvantages over the others. ${ }^{9}$ CHROMagar (CMRSA) is a selective and differential medium used for early detection of MRSA within 24hrs from clinical specimens including nasal, peri-anal swabs and a sputum. ${ }^{10}$ MRSA strain grow in the presence of antibiotic (cefoxitin) and after hydrolysis of chromogenic substrate produces blue colored colonies. Growth of other microbes can be suppressed by the addition of selective agents. ${ }^{11}$ The aim of the current study was to determine the frequency of nasal carriage of MRSA amongst hospital population and comparison of CMRSA with conventional methods for its detection.

\section{Materials and methods}

\section{Materials}

Sterile wooden swab (Copan, Italy), CMRSA (Lab M, UK), Blood agar (Oxoid, UK), cefoxitin disc (Oxoid, UK) Mueller Hinton agar (MHA) (Ox`oid, UK), Microbank beads (Pro-Lab Diagnostics, UK).

\section{Standard control strain}

Staphylococcus aureus ATCC 25923 and MRSA ATCC 33591 (obtained from UHS Lahore).

\section{Exclusion criteria}

Subjects having treatment with intranasal anti-MRSA ointments and other antibiotics in the last 14 days were excluded from the study.

\section{Inclusion criteria}

Patients with more than 3 days of hospital stay, doctors and healthcare workers were included in the study. 


\section{Ethical issues}

Patients and controls were informed about the study and were explained that their samples were used for the research purpose. A written informed consent was taken from all of the participants before the collection of samples.

\section{Settings}

The samples were taken from the different wards of Sheikh Zayed Hospital, Lahore and the study period of six months was carried out at the Department of Microbiology, University of Health Sciences, Lahore Pakistan

\section{Sample collection and media preparation}

A total of 200 nasal samples for bacterial isolation were taken from the hospital staff as well as patients exceeding 3 days of the hospitalization with sterile wooden swab. CMRSA and blood agar plates were prepared according to manufacturers, instructions.

\section{Processing of samples}

All samples were separately and evenly spreaded over the plated media, CMRSA and blood agar. The inoculated plates were then incubated at $37^{\circ} \mathrm{C}$ and growth checked after $24 \mathrm{hr}$. CMRSA plates that were negative for any type of growth were given subsequent incubation of $24 \mathrm{hr}$. Working stock culture of the pure cultures thus obtained was maintained on nutrient agar slants and the pure strains were incubated with Microbank beads and stored at minus $70^{\circ} \mathrm{C}$.

\section{Characterization of the bacterial isolates}

For determination of morphological and biochemical characteristics, the bacteria were processed as described by Mackie and McCartney, 1996. Based upon their susceptibility to cefoxitin disc $(30 \mu \mathrm{g})$ in Mueller-Hinton agar plates with $4 \% \mathrm{NaCl}$, the bacterial isolates were identified as MRSA after measuring the zone diameter. Zone size $<21 \mathrm{~mm}$ was considered to be resistant and zone size $>22 \mathrm{~mm}$ was considered to be sensitive according to the CLSI 2010 recommendations.

\section{Statistical analysis}

The data was analyzed using SPSS (19.0). Frequencies, percentages and graphs were given for qualitative variables. Diagnostic statistics like sensitivity, specificity, positive predictive value (PPV), negative predictive value (NPV) and diagnostic accuracy were calculated.

\section{Results}

A total of 200 nasal samples were taken from the indoor patients exceeding 3 days of hospitalization irrespective of the age and sex. Samples collected from different wards of healthcare unit (Figure 1). Out of total samples, 142(71\%) were susceptible to cefoxitin $(30 \mu \mathrm{g})$, and were methicillin sensitive Saureus (MSSA). The 45 samples were inoculated on CMRSA and blood agar simultaneously. Of the total MRSA isolated, one was obtained from hospital staff and the rest 44 were isolated from the patients. 7 samples were taken from Nephrology ward out of which 5 samples $(71.4 \%)$ were positive for MRSA. Among 45 samples, a total of 21(10.5\%) and 24(22.5\%) samples showed positive results for MRSA after 24 and subsequent $48 \mathrm{~h}$ of incubation at $37^{\circ} \mathrm{C}$ with blue colored colonies on CMRSA agar plates (Figure 2) while after $24 \mathrm{hr}$ of incubation at $37^{\circ} \mathrm{C}$ on blood agar utilizing cefoxitin disc diffusion method the same shown resistance and identified as MRSA. An increase in sensitivity was noted for CMRSA from $46.7 \%$ upto $100 \%$ upon twice incubation one after the other (Figures 2) (Figure 3). ${ }^{12}$ Blue colonies on CMRSA were identified as coagulase negative Staphylococci (CoNs). The organisms were identified as S. epidermidis and were susceptible to novobicin disc $(30 \mu \mathrm{g})$. Overall sensitivity of CMRSA was noted $100 \%$ when excluding blue colonies that were coagulase negative or had a gram stain not consistent with MRSA after $48 \mathrm{hr}$ of incubation.

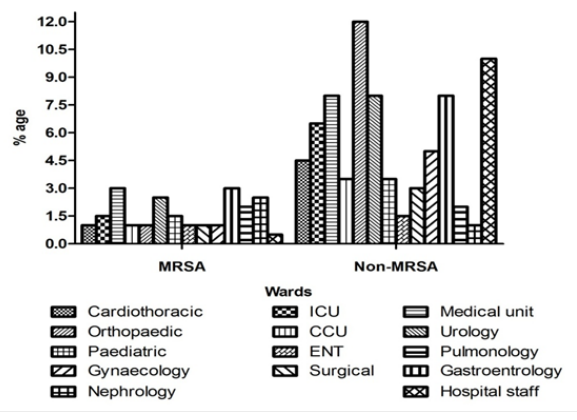

Figure I Comparison of Percentages of Methicillin-resistant Staphylococcus aureus (MRSA) with Staphylococci other than MRSA (Non-MRSA) from different wards of hospital.

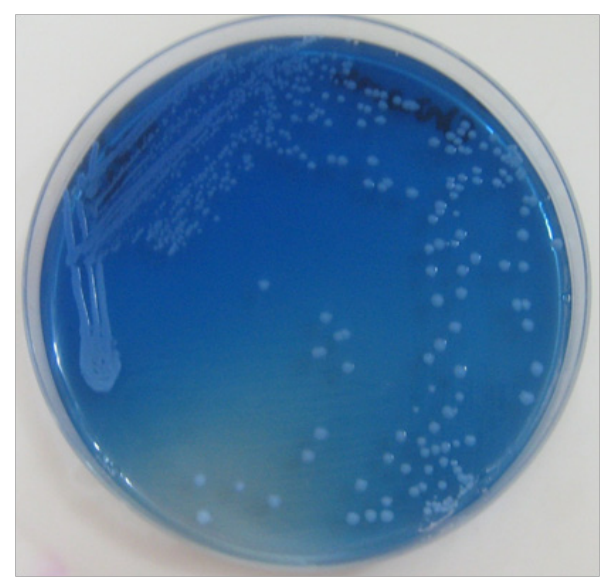

Figure 2 Plate of CHROMagar MRSA showing blue colored colonies of MRSA.

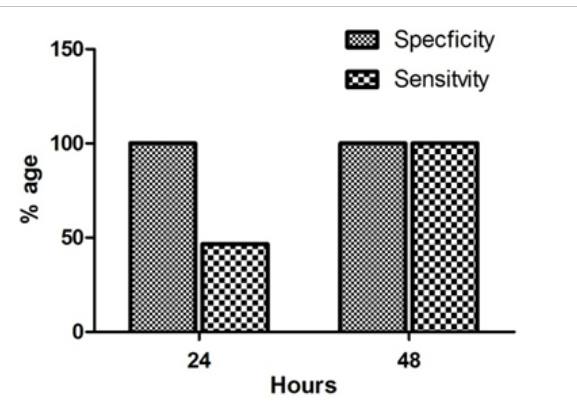

Figure 3 Comparison of specificity and sensitivity of MRSA in CHROM-agarMRSA (CMRSA) after 24 and $48 \mathrm{~h}$ of incubation.

\section{Discussion}

Detection of MRSA from clinical samples is of crucial importance for choosing appropriate antimicrobial therapy and to control MRSA endemicity. ${ }^{13}$ The present study was aimed to find out the rapid, reliable, cost-effective and easily applicable method for MRSA 
detection in routine microbiology laboratory. CMRSA was found to have improved sensitivity and specificity for the recovery of MRSA within $24-48 \mathrm{hrs}$. It was also observed that negative cultures (at 24hrs) when reincubated for another 24hrs, grew 24 more MRSA (Figure 1). CMRSA gave $100 \%$ specificity and sensitivity to detect MRSA when compared to that of conventional media. ${ }^{14}$ Stoakes et al. ${ }^{11}$ found that only $3 \%$ of colored colonies at $24 \mathrm{hrs}$ gave false-positive results on CMRSA but they were not MRSA whereas in the present study, $6.5 \%$ of colored colonies were found to be coagulase negative Staphylococci i.e. Methicllin resistant Staphylococcus epidermidis (MRSE). ${ }^{11}$ Vaerenbergh et al. ${ }^{14}$ found CMRSA to be $98.9 \%$ sensitive and $89.4 \%$ specific at $48 \mathrm{hrs} .{ }^{15}$ The results of the above mentioned studies are in accordance with the present study. On the basis of the results, it is suggested that the blue colored colonies on CMRSA must be carefully examined by gram staining, catalase, coagulase and other biochemical tests. The cost of one plate of CMRSA is more than that of Mueller-Hinton agar plates with cefoxitin disc.

However, identification of other colonies that grew on blood agar plates, need further subculturing and additional biochemical tests. So culturing the specimen on CMRSA from clinical specimens remains economical. ${ }^{14}$ In Pakistan, several independent studies regarding the frequency of MRSA have been reported that show a remarkable difference. In our present study, $22.5 \%$ were MRSA, $71 \%$ were MSSA and the rest $6.5 \%$ were MRSE. Bukhari et al. ${ }^{16}$ reported $27.9 \%$ of their isolates as MRSA of all $\mathrm{S}$. aureus isolates in a study carried out in King Edward Medical University, Lahore. ${ }^{16}$ Khatoon et al reported $38.5 \%$ prevalence of MRSA infections in a study carried out at Mayo Hospital Lahore in 2000. ${ }^{17}$ According to another study at Military Hospital Rawalpindi (2006), 42.01\% of all nasal staphylococci were MRSA. The samples were taken after $72 \mathrm{hrs}$ of admission. None of the patient had MRSA infection at the admission time. ${ }^{18}$ This observation clearly indicates that there is a need to screen all the indoor patients if they stay more than $48 \mathrm{hr}$ in the hospital. There is a difference in MRSA isolation frequency in developed parts of world. According to one study conducted in Chicago (2005), 7.9\% of all staphylococci were MRSA with high isolation rate from nasal site sample (84\% of total isolates). ${ }^{19,20}$ This low frequency is due to their implementation of infection control awareness programmes such as hand washing, gloves, gowns, masks, isolation of MRSA patients and use of suitable disinfectants in clinical practice. It is important that antimicrobial policy should promote that rational use of antibiotics.

\section{Conclusion}

CMRSA is useful in the rapid identification of MRSA in 24-48 hrs in a single step directly from clinical specimens thereby reducing detection time without additional susceptibility testing, enhanced recovery of MRSA, suppression of MSSA and other non-MRSA species that might be present in the nose.

\section{Recommendations}

Proper precautionary measures should be followed to prevent MRSA infection. Implementation of infection control awareness programmes such as hand washing, gloves, gowns, masks, isolation of MRSA patients and use of suitable disinfectants in clinical use will help to reduce the risk factors that associated with the emergence of multidrug resistance. Yearly data should be collected from different hospitals to observe the prevalence of MRSA.

\section{Acknowledgment}

We acknowledge our gratitude to University of Health Sciences, Lahore for fully supporting this research activity. We are extremely thankful to Sheikh Zayed Hospital, Lahore for providing clinical samples. We also thank Mr. Muhammad Yasir, Mr. Usman, Mr. Abdul Quddus Tariq, and Mr. Wasim Akhtar for their valuable technical assistance and unconditional support.

\section{Conflict of interest}

The author declares no conflict of interest.

\section{References}

1. Foster TJ. The Staphylococcus aureus superbug. J Clin Invest. 2004;114(2):1693-1696.

2. De San N, Denis O, Gasasira MF, et al. Controlled evaluation of the IDI-MRSA assay for detection of colonization by methicillin-resistant Staphylococcus aureus in diverse mucocutaneous specimens. J Clin Microbiol. 2007;45(4):1098-1101.

3. Bukhari $\mathrm{MH}$, Iqbal A, Khatoon $\mathrm{N}$, et al. A laboratory study of susceptibility of methicillin resistant staphylococcus aureus (MRSA). Pak J Med Sci. 2004;20(3):229-233.

4. Skiest DJ, Brown K, Cooper TW, Prospective comparison of methicillin-susceptible and methicillin-resistant community-associated Staphylococcus aureus infections in hospitalized patients. J Infect. 2007;54(5):427-434

5. Jones ME, Karlowsky JA, Draghi DC, et al. Epidemiology and antibiotic susceptibility of bacteria causing skin and soft tissue infections in the USA and Europe: a guide to appropriate antimicrobial therapy. Int $J$ Antimicrob Agents. 2003;22(4):406-419.

6. Flayhart D, Hindler J, Bruckner DA, et al. Multicenter evaluation of BBL CHROMagar MRSA medium for direct detection of methicillinresistant $\mathrm{S}$. aureus from surveillance cultures of the anterior nares. J Clin Microbiol. 2005;43(11):5536-5540.

7. Naimi TS, LeDell KH, Como-Sabetti K, et al. Comparison of communityand health care-associated methicillin-resistant staphylococcus aureus infection. JAMA. 2003;290(22):2976-284.

8. Taj R, Muhammadzai I, Ahmad J, et al. Frequency and antibiotic susceptibility pattern of methicillin-resistant staphylococcus aureus in abbottabad city of Pakistan. KMUJ. 2015;7(4):1-5.

9. Eigner U, Weizenegger M, Fahr M, et al. Evaluation of a rapid direct assay for identification of bacteria and the mecA and van genes from positive-testing blood cultures. J Clin Microbiol. 2005;43(10):52565262 .

10. Pape J, Wadlin J, Nachamkin I. Use of BBL CHROMagar MRSA medium for Identification of MRSA directly from blood cultures. J Clin Microbiol. 2006;44:2575-76.

11. Stoakes L, Reyes R, Daniel J, et al. Prospective comparison of a new chromogenic medium, MRSA Select, to CHROMagar MRSA and mannitol-salt medium supplemented with oxacillin or cefoxitin for detection of MRSA. J Clin Microbiol. 2006;44(2):637-639.

12. Winn WC, Allen SD, Janda WM, et al. Charts. In: Lippincott Williams, et al. editors. 6th ed. Koneman's Color Atlas and Textbook of Diagnostic Microbiology. India: Lippincott Williams, Wilkins; 2006. p. 1442-1535.

13. Grundmann H, Aires-de-Sousa M, Boyce J, et al. Emergence and resurgence of methicillin-resistant Staphylococcus aureus as a publichealth threat. Lancet. 2006;368(9538):874-885.

14. Taguchi H, Kaneko T, Onozaki M, Kubo R, Kamiya S. CHROMagar MRSA-evaluation of a new chromogenic medium for isolation of MRSA. J Jap Assoc Infect Dis. 2004. p. 54-58.

15. Vaerenbergh KV, Cartuyvels R, Coppens G, et a. Performance of a new chromogenic medium, BBL CHROMagar MRSA II (BD), for detection of methicillin-resistant staphylococcus aureus in screening samples. $J$ Clin Microbiol. 2010;48(4):1450-1451. 
16. Bukhari SZ, Ahmed S. Prevalence of methicillin resistance among Staphylococcus aureus isolates in Pakistan and its clinical outcome. $J$ Hosp Infect. 2007;67(1):101-102.

17. Khatoon N, Bukhari MH, Raja RJ. Prevalence of Methicillin resistant staphylococcus aureus (MRSA) infection: Laboratory study at Mayo Hospital Lahore. Biomedica. 2002;18:49-52.

18. Ali AM, Abbasi SA, Arif S, et al. Nosocomial infections due to Methicillin Resistant Staphylococcus aureus (MRSA) in hospitalized patients. Pak J Med Sci. 2007;23:593-596.
19. Lucet JC, Grenet K, Lefevre LA, et al. High prevalence of MRSA at hospital admission in elderly patients: Implication for infection control strategies. Infect Control Hosp Epidemiol. 2005;26(2):120-126.

20. Duguid JP. Staining Methods. In: Collee JG, et al. editors. Mackie and McCartney Practical Medical Microbiology 14th ed. India: Reed India Elsevier Private Limited; 1996. p. 793-812. 